\title{
The reliability of shear elastic modulus measurement of the ankle plantar flexion muscles is higher at dorsiflexed position of the ankle
}

\author{
Junya Saeki ${ }^{1,2^{*}}$ D, Tome Ikezoe ${ }^{1}$, Masatoshi Nakamura ${ }^{3}$, Satoru Nishishita ${ }^{1,4,5}$ and Noriaki Ichihashi ${ }^{1}$
}

\begin{abstract}
Background: Excessive stiffness of lower limb muscles is associated with sports injuries. Therefore, the identification of a reliable measurement of the shear elastic modulus of various ankle plantar flexion muscles is required to evaluate lower leg sports injuries. This study investigated the reliable measurement of the shear elastic modulus of the ankle plantar flexion muscles at different ankle positions.

Methods: Twenty-three healthy young men $(25.3 \pm 3.6$ years, $172.9 \pm 5.0 \mathrm{~cm}, 67.2 \pm 7.2 \mathrm{~kg})$ participated in this study. The shear elastic moduli of the ankle plantar flexion muscles including the lateral gastrocnemius, medial gastrocnemius, soleus, peroneus longus, peroneus brevis, flexor hallucis longus, flexor digitorum longus and tibialis posterior were measured using ultrasonic shear wave elastography at 0,10 and $20^{\circ}$ dorsiflexion.

Results: The reliability of the shear elastic modulus measurements for each ankle position was assessed. The results showed that the interday reliability of the measurements differed between ankle positions and that the reliability of the shear elastic modulus measurements at $20^{\circ}$ dorsiflexion was higher than that at $10^{\circ}$ or $0^{\circ}$.
\end{abstract}

Conclusion: Our results suggest that measurements at $20^{\circ}$ dorsiflexion may provide a more reliable measurement of the shear elastic modulus of ankle plantar flexion muscles.

Keywords: Shear-wave elastography, Shear elastic modulus, Muscle stiffness, Muscle hardness, Reliability, Tibialis posterior, Flexor digitorum longus, Flexor hallucis longus

\section{Background}

It has been reported that an overuse injury in sports is associated with soft tissue stiffness $[1,2]$. In particular, the elongational stress of lower limb muscles such as the soleus (SOL), flexor digitorum longus (FDL) or tibialis posterior (TP) increases the strain in the tibial fascia [3]. These muscles have been identified as risk factors for medial tibial stress syndrome (MTSS), which commonly develops in runners. In addition, it is considered that excessive stiffness of the lower limb muscles is also associated with MTSS [4].

\footnotetext{
* Correspondence: saeki.junya.55z@kyoto-u.jp

${ }^{1}$ Human Health Sciences, Graduate School of Medicine, Kyoto University, 53

Shogoin-Kawahara-cho, Sakyo-ku, Kyoto 606-8507, Japan

${ }^{2}$ Research Fellow of the Japan Society for the Promotion of Science, 5-3-1

Kojimachi, Chiyoda-ku, Tokyo 102-0083, Japan

Full list of author information is available at the end of the article
}

Therefore, the assessment and intervention of lower limb muscle stiffness are important for preventing MTSS.

In recent years, it has become possible to evaluate muscle stiffness quantitatively in vivo with the shear elastic modulus, which is calculated from the velocity of the shear wave created by the vibration of tissue using shear wave elastography [5-7]. Regarding the shear elastic modulus of the medial gastrocnemius (MG), lateral gastrocnemius (LG) and SOL using shear wave elastography, previous studies have shown its availability as an index of muscle stiffness $[4,8]$. Other previous studies also demonstrated the reliability of shear elastic modulus measurements of the MG, LG and SOL $[9,10]$. Although the shear elastic modulus of the triceps surae has been considered as a useful index of muscle stiffness, the reliability of shear elastic modulus measurements of other 
ankle plantar flexion muscles remains unclear. However, the muscle stiffnesses of the LG, MG, SOL and peroneus longus (PL) were higher in patients with MTSS [4]. Therefore, the identification of a reliable measurement of the shear elastic modulus of various ankle plantar flexion muscles is required to evaluate lower leg sports injuries. The purpose of this study was to investigate the reliability of the shear elastic modulus of the ankle plantar flexion muscles at different ankle positions and to determine the ankle position that can provide the most reliable measurement.

\section{Methods}

\section{Subjects}

Twenty-three healthy young men participated in this study. The mean values ( \pm the standard deviation (SD)) for their age, height and mass were $25.3 \pm 3.6$ years, 172.9 $\pm 5.0 \mathrm{~cm}$ and $67.2 \pm 7.2 \mathrm{~kg}$, respectively. Subjects with a history of neuromuscular disease or musculoskeletal injury on the lower limbs were excluded. All participants had a maximum ankle dorsiflexion (DF) angle of at least $20^{\circ}$. Participants were given precise information about the content and order of the study. In addition, informed consent was obtained from all participants. This study was approved by the ethics committee of Kyoto University Graduate School and the Faculty of Medicine (R0266).

\section{Procedures}

\section{Measurements of shear elastic modulus}

The shear elastic modulus of a muscle was measured using ultrasonic shear wave elastography (Aixplorer, Supersonic Imagine, France) with a linear array probe (SL10-2, Supersonic Imagine, France). The region of interest (ROI) was set near the centre of each muscle. Adopting the method used in a previous study [11], the shear elastic modulus $\mu$ was calculated as follows:

$$
\mu(k P a)=\rho V s^{2}
$$

where Vs is the shear wave velocity, and $\rho$ is the muscle mass density $\left(1000 \mathrm{~kg} \cdot \mathrm{m}^{-3}\right)$. The analysis area was a 5 mm-diameter circle at the centre of the ROI [12]. The shear elastic modulus was measured three times per muscle, and the average of the three measured values was used for further analysis. To assess the reliability, the same protocol was performed on two different days by the same investigator at an interval of two weeks. The measurement of shear elastic modulus was performed by the same observer (JS) with at least $100 \mathrm{~h}$ of experience in ultrasound elastography. Considering the intraday fluctuation in the shear elastic modulus caused by muscle fatigue, the time of measurement for the interday analysis was matched as much as possible.

The target muscles were MG, LG, SOL, PL, peroneus brevis (PB), flexor halluces longus (FHL), FDL, and TP.
These muscles have the moment arm of plantar flexion of the ankle [13]. The shear elastic moduli of the LG, MG, SOL, PL, PB, and FHL were measured in a prone position, while those for the FDL and TP were measured in a supine position. The shear elastic moduli of the LG, MG and SOL were measured at proximal $30 \%$ of the lower leg length from the popliteal crease to the lateral malleolus [8, 14]. The shear elastic moduli of the PL and PB were measured at proximal $30 \%$ from the head of the fibula to the lateral malleolus. The shear elastic modulus of the FHL was measured at proximal $60 \%$ from the head of the fibula to the lateral malleolus. The shear elastic modulus of the FDL was measured at proximal 50\% from the cleavage line of the knee joint to the medial malleolus. The shear elastic modulus of the TP was measured at proximal $40 \%$ from the cleavage line of the knee joint to the medial malleolus for the superficial layer of the intramuscular tendon and at proximal $60 \%$ from the cleavage line of the knee joint to the medial malleolus for the deep layer of the intramuscular tendon (Figs. 1 and 2). These locations were

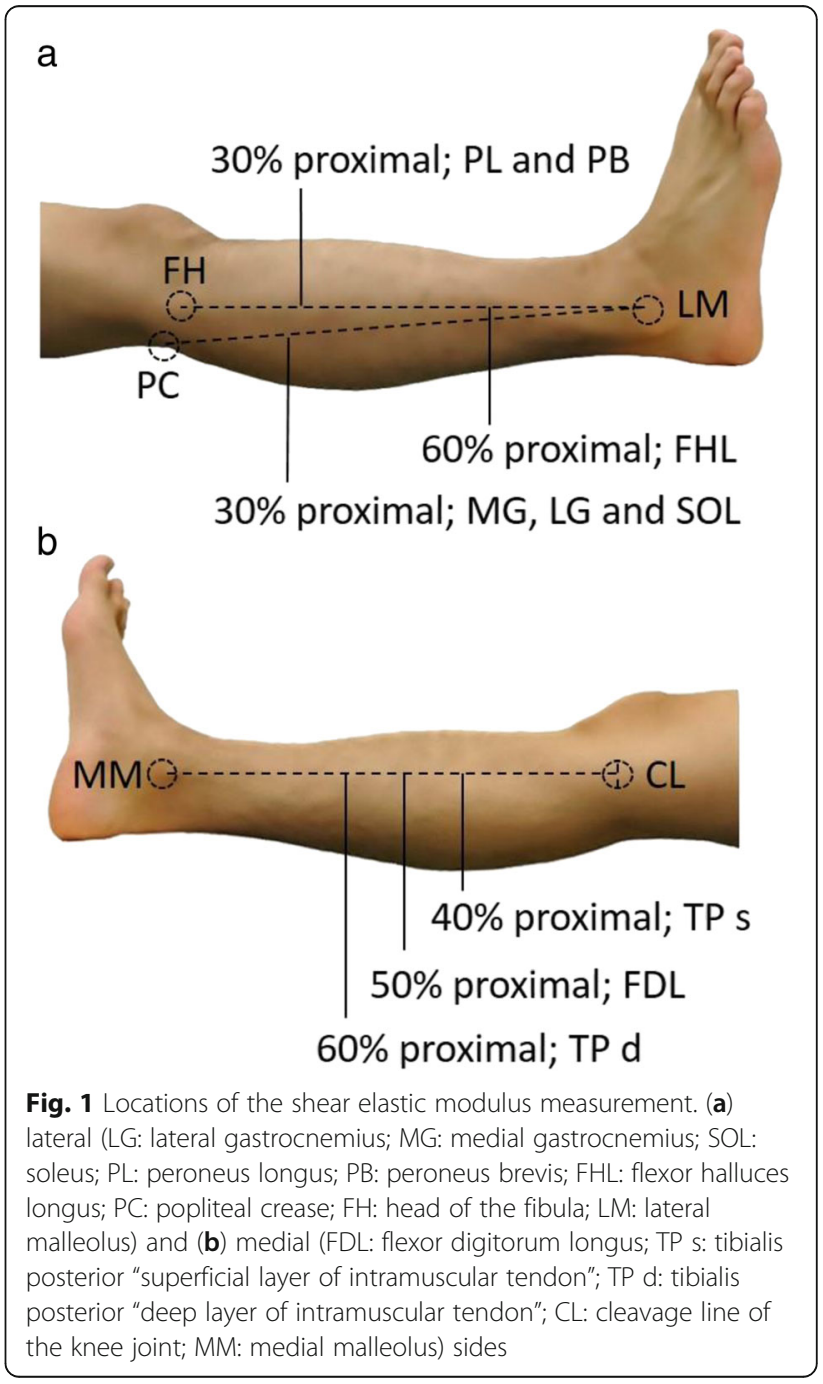




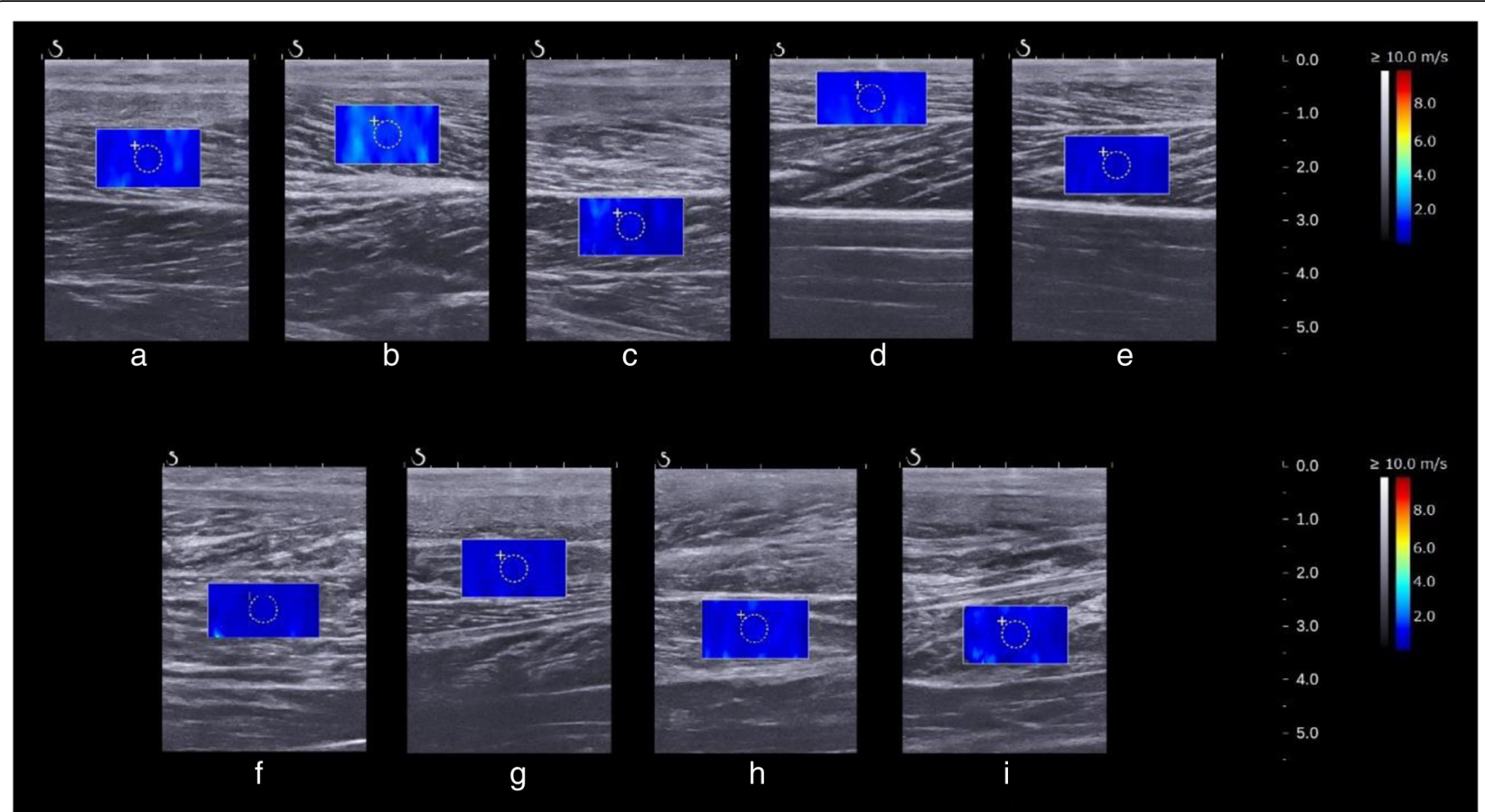

Fig. 2 Ultrasound images of shear elastic modulus measurement. Ultrasound images of the shear elastic modulus measurement (a) lateral gastrocnemius (b) medial gastrocnemius (c) soleus (d) peroneus longus (e) peroneus brevis (f) flexor hallucis longus (g) flexor digitorum longus (h) tibialis posterior (superficial layer of intramuscular tendon) (i) tibialis posterior (deep layer of intramuscular tendon)

identified by confirming movement of muscle fibers in a B-mode ultrasound image during passive movement of the ankle or toes.

The subjects laid on an electrodynamometer (Biodex System 4, Biodex, USA) with the knee in full extension and the ankle joint securely attached to the footplate of the dynamometer. They were instructed to remain relaxed during the measurements. Ultrasound images were recorded along the longitudinal axis of the muscle at ankle DF of 0,10 and $20^{\circ}$. The examiner checked whether the heel was fixed securely to the footplate of the dynamometer during measurement. The footplate being perpendicular to the fibula was defined as the $0^{\circ}$ dorsiflexion of the ankle joint. The same procedure was followed on day 1 and day 2, and the setting of BIODEX was identical on both days. Measurements of each ankle position were performed in the order mentioned, i.e. 0 , 10 and $20^{\circ} \mathrm{DF}$, to reduce the effect of stretching.

\section{Statistical analysis}

Statistical analyses were performed using statistical software (SPSS Statistics 22, IBM, USA). The intra-class correlation coefficient (ICC) (1.3) using the average value of each day was assessed for the analyses of the interday reliability. In addition, the coefficient of variation (CV) and the standard error of the mean (SEM) were calculated. The $\mathrm{CV}$ was calculated by dividing the
$\mathrm{SD}$ of repeated measurements by the average value, while the SEM was calculated by dividing the SD of repeated measurements by the number of measurements (that is, six times).

A one-way repeated measures analysis of variance (ANOVA) with Bonferroni's post hoc test were used to determine the differences in the shear elastic moduli between ankle positions. For all tests, the statistical significance was set at $p<0.05$. On the basis of previous studies [10, 15], the reliability was defined as almost perfect if the ICC was greater than 0.81 and the $C V$ was less than $10 \%$.

\section{Results}

The results of the reliability are shown in Table 1 . The ICCs ranged from 0.58 to 0.83 for $0^{\circ} \mathrm{DF}, 0.65$ to 0.87 for $10^{\circ} \mathrm{DF}$, and 0.71 to 0.95 for $20^{\circ} \mathrm{DF}$. The $\mathrm{CV}$ ranged from 6.5 to $12.0 \%$ for $0^{\circ} \mathrm{DF}, 5.7$ to $11.9 \%$ for $10^{\circ} \mathrm{DF}$, and 4.7 to $13.6 \%$ for $20^{\circ} \mathrm{DF}$. The SEM ranged from 0.2 to 0.4 $\mathrm{kPa}$ for $0^{\circ} \mathrm{DF}, 0.2$ to $0.6 \mathrm{kPa}$ for $10^{\circ} \mathrm{DF}$, and 0.2 to 1.4 $\mathrm{kPa}$ for $20^{\circ} \mathrm{DF}$.

The differences in the shear elastic moduli between ankle positions are summarized in Table 2. The one-way repeated measures ANOVA indicated significant main effects of the ankle position for all muscles. The post hoc analysis showed significant differences between all ankle positions, which shows that the shear elastic modulus increases with the ankle DF for all muscles. 
Table 1 Reliabilities of the shear elastic modulus measurements

\begin{tabular}{|c|c|c|c|c|c|c|c|c|c|}
\hline & LG & MG & $\mathrm{SOL}$ & $\mathrm{PL}$ & PB & $\mathrm{FHL}$ & $\mathrm{FDL}$ & TP s & $\mathrm{TP} d$ \\
\hline \multicolumn{10}{|l|}{ DF 0} \\
\hline Shear elastic modulus of day $1(\mathrm{kPa})$ & 8.7 & 12.3 & 4.6 & 7.0 & 5.7 & 4.6 & 6.0 & 7.3 & 7.7 \\
\hline Shear elastic modulus of day2 ( $\mathrm{kPa})$ & 8.4 & 11.8 & 4.7 & 7.1 & 5.9 & 4.9 & 6.3 & 7.9 & 8.0 \\
\hline Mean value of day 1 and day $2(\mathrm{kPa})$ & 8.5 & 12.1 & 4.6 & 7.1 & 5.8 & 4.8 & 6.1 & 7.6 & 7.8 \\
\hline $\mathrm{SD}(\mathrm{kPa})$ & 1.7 & 2.7 & 1.1 & 1.7 & 1.0 & 1.2 & 1.4 & 2.0 & 1.8 \\
\hline SEM (kPa) & 0.2 & 0.3 & 0.2 & 0.2 & 0.2 & 0.2 & 0.2 & 0.3 & 0.4 \\
\hline CV (\%) & 6.5 & 6.5 & 12.0 & 7.6 & 8.5 & 9.2 & 8.4 & 8.9 & 11.3 \\
\hline ICC (1.3) & 0.77 & 0.76 & 0.67 & 0.80 & 0.58 & 0.76 & 0.78 & 0.83 & 0.67 \\
\hline \multicolumn{10}{|l|}{ DF 10} \\
\hline Shear elastic modulus of day $1(\mathrm{kPa})$ & 16.1 & 25.5 & 6.4 & 9.2 & 7.2 & 6.0 & 7.2 & 8.9 & 9.3 \\
\hline Shear elastic modulus of day2 ( $\mathrm{kPa})$ & 15.3 & 24.8 & 6.2 & 8.7 & 7.4 & 6.2 & 7.4 & 8.7 & 9.1 \\
\hline Mean value of day 1 and day $2(\mathrm{kPa})$ & 15.7 & 25.1 & 6.3 & 8.9 & 7.3 & 6.1 & 7.3 & 8.8 & 9.2 \\
\hline $\mathrm{SD}(\mathrm{kPa})$ & 3.0 & 5.5 & 1.5 & 2.2 & 1.7 & 1.5 & 1.4 & 2.6 & 2.6 \\
\hline $\mathrm{SEM}(\mathrm{kPa})$ & 0.4 & 0.6 & 0.3 & 0.3 & 0.2 & 0.2 & 0.2 & 0.3 & 0.4 \\
\hline CV (\%) & 6.7 & 5.7 & 9.6 & 7.5 & 8.2 & 7.0 & 7.7 & 8.9 & 11.9 \\
\hline ICC (1.3) & 0.76 & 0.80 & 0.80 & 0.82 & 0.72 & 0.87 & 0.65 & 0.83 & 0.76 \\
\hline \multicolumn{10}{|l|}{ DF 20} \\
\hline Shear elastic modulus of day $1(\mathrm{KPa})$ & 31.5 & 53.7 & 10.3 & 11.6 & 9.5 & 8.8 & 9.4 & 11.4 & 11.5 \\
\hline Shear elastic modulus of day2 ( $\mathrm{kPa})$ & 32.2 & 53.4 & 10.8 & 12.3 & 9.9 & 8.7 & 9.4 & 11.5 & 11.7 \\
\hline Mean value of day 1 and day $2(\mathrm{kPa})$ & 31.8 & 53.6 & 10.5 & 12.0 & 9.7 & 8.7 & 9.4 & 11.5 & 11.6 \\
\hline $\mathrm{SD}(\mathrm{kPa})$ & 6.6 & 11.5 & 3.2 & 4.3 & 2.8 & 2.3 & 2.5 & 3.9 & 3.5 \\
\hline SEM (kPa) & 0.6 & 1.4 & 0.4 & 0.3 & 0.3 & 0.2 & 0.2 & 0.3 & 0.6 \\
\hline CV (\%) & 4.7 & 6.3 & 9.4 & 7.1 & 7.9 & 6.6 & 6.2 & 5.6 & 13.6 \\
\hline ICC (1.3) & 0.91 & 0.82 & 0.81 & 0.92 & 0.85 & 0.86 & 0.91 & 0.95 & 0.71 \\
\hline
\end{tabular}

$S D$ standard deviation, SEM standard error in measurement, $C V$ coefficient of variation, ICC intra-class correlation coefficient, $L G$ lateral gastrocnemius, $M G$ medial gastrocnemius, $S O L$ soleus, $P L$ peroneus longus, $P B$ peroneus brevis, FHL flexor hallucis longus, FDL flexor digitorum longus, $T P$ s tibialis posterior (superficial layer of intramuscular tendon), TP $d$ tibialis posterior (deep layer of intramuscular tendon)

Table 2 The differences in the shear elastic moduli between ankle positions

\begin{tabular}{lccc}
\hline \multicolumn{4}{l}{ Shear elastic modulus (kPa) } \\
\hline
\end{tabular}

The data has been expressed as the mean +/- Standard deviation of the values observed on day 1 and day 2 . The data has been expressed as the mean of the values observed on day 1 and day 2

\section{Discussion}

This study focused on the ankle plantar flexion muscles and investigated the reliability of shear elastic modulus measurements. To the best of our knowledge, this is the first study examining the shear elastic moduli of the $\mathrm{PB}$, FHL, FDL and TP using shear wave elastography at different ankle positions. The results showed that the CV for the LG, MG and SOL were 4.7 to $6.7 \%, 5.7$ to $6.5 \%$ and 9.4 to $12.0 \%$, respectively, which were lower than the CV values of the previous study (9 to $15 \%, 9 \%$, and 19 to $20 \%$, respectively) [9]. This is probably due to the ankle angle during measurement, which was set more accurately in our study, since it was securely attached to the footplate of the dynamometer.

As for the reliability of the superficial TP, the ICCs were greater than 0.81 , and the CVs were less than $10 \%$ at all ankle positions, which confirmed the high reliability of the shear elastic modulus measurements of the superficial TP at any ankle positions from 0 to $20^{\circ} \mathrm{DF}$. 
On the other hand, the ICCs of the PL and FHL were less than 0.81 , and the CVs were less than $10 \%$ at $0^{\circ} \mathrm{DF}$, whereas the ICCs were greater than 0.81 at $10^{\circ}$ and $20^{\circ}$ DF. Furthermore, for the LG, MG, SOL, PB and FDL, the ICCs were greater than 0.81 , and the CVs were less than $10 \%$ only at $20^{\circ} \mathrm{DF}$. These results suggest that high reliability may be attained for shear elastic modulus measurement at 10 and $20^{\circ} \mathrm{DF}$ for the PL and FHL and at $20^{\circ} \mathrm{DF}$ for the LG, MG, SOL, PB and FDL.

Our findings suggest that the reliability of the shear elastic modulus measurements differed between ankle positions. The one-way repeated measures ANOVA and post hoc analysis showed that there were significant effects of the ankle positions on the shear elastic modulus for all muscles and that the shear elastic modulus of all muscles increased with ankle DF. These results suggest that the ankle plantar flexion muscles, which were measured in this study, were elongated with ankle DF. The results of this study showed that the ICC at $0^{\circ} \mathrm{DF}$ was 0.67 for the SOL, which was lower compared to the ICCs of the LG and MG. The previous study had reported that the slack angles (i.e. the joint angle corresponding to the muscle length beyond which the muscle begins to develop a passive force) of the LG, MG and SOL were $14.9 \pm 6.7^{\circ}$ for plantar flexion, $20.7 \pm 6.7^{\circ}$ for plantar flexion and $2.0 \pm 4.8^{\circ}$ for the DF position, respectively [16]. These results suggested that the slack angle of the SOL was greater in the DF direction than those of the LG and MG. Therefore, it is likely that the shear elastic modulus of the SOL measured at $0^{\circ}$ did not reflect the subject characteristics. In addition, it was reported that the ICC is affected by the dispersion of the intersubject measured values [17]. It was considered that the low ICC for the SOL was calculated at $0^{\circ} \mathrm{DF}$ owing to the small SD. For the same reason, lower ICCs may have been calculated for some muscles at $0^{\circ}$ or $10^{\circ} \mathrm{DF}$.

The ICC for the deep TP was less than 0.81, and the CVs were greater than $10 \%$ at any ankle position. The possible reason for the lower ICC and higher $\mathrm{CV}$ of the deep TP is the unstable value measured because the shear wave did not reach the target tissue owing to the effect of the superficial tissue (e.g. the SOL, FDL and fascia). On the other hand, the ICCs for the superficial TP were greater than 0.81 , and the CVs were less than $10 \%$ at all ankle positions. These results suggested that the shear elastic modulus of the TP could be measured more reliably at the proximal superficial layer than at the distal deep layer.

In this study, there was a limitation in that we did not investigate the interrater reliability. Therefore, we were not able to determine whether the shear elastic modulus of a newly measured muscle is comparable among different testers or not. In addition, measurement of the shear elastic modulus also depends on the skill of the tester
[18]. Therefore, testers need to practice before taking measurements until a high reliability is obtained, even if a high reliability was shown in this study.

\section{Conclusions}

This study investigated the reliability of shear elastic modulus measurement for the ankle plantar flexion muscles at different ankle positions. The results of this study showed that the measurement reliability of the shear elastic modulus differed between ankle positions and that a high reliability was observed when measuring the shear elastic moduli of the TP at 0 to $20^{\circ} \mathrm{DF}$; the PL and FHL at 10 to $20^{\circ} \mathrm{DF}$; and the LG, MG, SOL, PB and $\mathrm{FDL}$ at $20^{\circ} \mathrm{DF}$. These results suggest that the shear elastic modulus of the ankle DF muscle should be measured at $20^{\circ} \mathrm{DF}$.

\section{Abbreviations \\ ANOVA: Analysis of variance; CV: Coefficient of variation; DF: Dorsiflexion; FDL: Flexor digitorum longus; FHL: Flexor hallucis longus; ICC: Intra-class correlation coefficient; LG: Lateral gastrocnemius; MG: Medial gastrocnemius; MTSS: Medial tibial stress syndrome; PB: Peroneus brevis; PL: Peroneus longus; ROI: Region of interest; SD: Standard deviation; SEM: Standard error of the mean; SOL: Soleus; TP: Tibialis posterior}

\section{Acknowledgments}

We appreciate Ms. Ibuki for checking the manuscript

\section{Funding}

This work was supported by a Grant-in-Aid from the Japan Society for the Promotion of Science Fellows (16 J11346).

\section{Availability of data and materials}

The data that support the findings of this study are available from the corresponding author upon reasonable request.

\section{Authors' contributions}

JS, MN, and NI devised and designed the experiments. JS performed the experiments and data analysis and drafted the manuscript. TI, MN, SN, and $\mathrm{NI}$ contributed to discussion and review of the manuscript. All authors read and approved the final manuscript prior to the submission.

\section{Competing interests}

The authors declare that they have no competing interests.

\section{Consent for publication}

Not applicable.

\section{Ethics approval and consent to participate}

Informed consent was obtained from all subjects. This study was approved by the ethics committee of the Kyoto University Graduate School and the Faculty of Medicine (approval number: R0266).

\section{Publisher's Note}

Springer Nature remains neutral with regard to jurisdictional claims in published maps and institutional affiliations.

\section{Author details}

${ }^{1}$ Human Health Sciences, Graduate School of Medicine, Kyoto University, 53 Shogoin-Kawahara-cho, Sakyo-ku, Kyoto 606-8507, Japan. ${ }^{2}$ Research Fellow of the Japan Society for the Promotion of Science, 5-3-1 Kojimachi, Chiyoda-ku, Tokyo 102-0083, Japan. ${ }^{3}$ Institute for Human Movement and Medical Sciences, Niigata University of Health and Welfare, Shimami-cho 1398, Kita-ku, Niigata 950-3198, Japan. ${ }^{4}$ Institute of Rehabilitation Science,

Tokuyukai Medical Corporation, 3-11-1 Sakurano-cho, Toyonaka, Osaka 
560-0054, Japan. ${ }^{5}$ Kansai Rehabilitation Hospital, 3-11-1 Sakurano-cho, Toyonaka, Osaka 560-0054, Japan.

Received: 20 December 2016 Accepted: 7 April 2017

Published online: 18 April 2017

\section{References}

1. Pamukoff DN, Blackburn JT. Comparison of plantar flexor musculotendinous stiffness, geometry, and architecture in male runners with and without a history of tibial stress fracture. J Appl Biomech. 2015;31:41-7.

2. Sconfienza $L M$, Silvestri E, Cimmino MA. Sonoelastography in the evaluation of painful Achilles tendon in amateur athletes. Clin Exp Rheumatol. 2010;28:373-8.

3. Bouche RT, Johnson CH. Medial tibial stress syndrome (tibial fasciitis): a proposed pathomechanical model involving fascial traction. J Am Podiatr Med Assoc. 2007;97:31-6.

4. Akiyama K, Akagi R, Hirayama K, Hirose N, Takahashi H, Fukubayshi T. Shear Modulus of the Lower Leg Muscles in Patients with Medial Tibial Stress Syndrome. Ultrasound Med Biol. 2016;42:1779-83.

5. Eby SF, Song P, Chen S, Chen Q, Greenleaf JF, An KN. Validation of shear wave elastography in skeletal muscle. J Biomech. 2013;46:2381-7.

6. Maisetti O, Hug F, Bouillard K, Nordez A. Characterization of passive elastic properties of the human medial gastrocnemius muscle belly using supersonic shear imaging. J Biomech. 2012;45:978-84.

7. Chernak LA, DeWall RJ, Lee KS, Thelen DG. Length and activation dependent variations in muscle shear wave speed. Physiol Meas. 2013;34:713-21.

8. Nakamura M, Ikezoe T, Kobayashi T, Umegaki H, Takeno Y, Nishishita S, Ichihashi N. Acute effects of static stretching on muscle hardness of the medial gastrocnemius muscle belly in humans: an ultrasonic shear-wave elastography study. Ultrasound Med Biol. 2014;40:1991-7.

9. Dubois $G$, Kheireddine W, Vergari C, Bonneau D, Thoreux P, Rouch P, Tanter M, Gennisson JL, Skalli W. Reliable protocol for shear wave elastography of lower limb muscles at rest and during passive stretching. Ultrasound Med Biol. 2015;41:2284-91.

10. Lacourpaille L, Hug F, Bouillard K, Hogrel JY, Nordez A. Supersonic shear imaging provides a reliable measurement of resting muscle shear elastic modulus. Physiol Meas. 2012;33:N19-28.

11. Gennisson JL, Deffieux T, Mace E, Montaldo G, Fink M, Tanter M. Viscoelastic and anisotropic mechanical properties of in vivo muscle tissue assessed by supersonic shear imaging. Ultrasound Med Biol. 2010;36:789-801.

12. Akagi $\mathrm{R}$, Takahashi $\mathrm{H}$. Effect of a 5 -week static stretching program on hardness of the gastrocnemius muscle. Scand J Med Sci Sports. 2014;24:950-7.

13. McCullough MB, Ringleb SI, Arai K, Kitaoka HB, Kaufman KR. Moment arms of the ankle throughout the range of motion in three planes. Foot Ankle Int. 2011;32:300-6.

14. Akagi R, Yamashita Y, Ueyasu Y. Age-Related Differences in Muscle Shear Moduli in the Lower Extremity. Ultrasound Med Biol. 2015;41:2906-12.

15. Landis JR, Koch GG. The measurement of observer agreement for categorical data. Biometrics. 1977;33:159-74.

16. Hirata K, Kanehisa H, Miyamoto-Mikami E, Miyamoto N. Evidence for intermuscle difference in slack angle in human triceps surae. J Biomech. 2015:48:1210-3

17. Hopkins WG. Measures of reliability in sports medicine and science. Sports Med. 2000;30:1-15.

18. Hirayama K, Akagi R, Takahashi H. Reliability of ultrasound elastography for the quantification of transversus abdominis elasticity. Acta Radiol Open. 2015:4:2058460115603420.

\section{Submit your next manuscript to BioMed Central and we will help you at every step:}

- We accept pre-submission inquiries

- Our selector tool helps you to find the most relevant journal

- We provide round the clock customer support

- Convenient online submission

- Thorough peer review

- Inclusion in PubMed and all major indexing services

- Maximum visibility for your research

Submit your manuscript at www.biomedcentral.com/submit 